\title{
Tensiomyography Variable Trend of Changes After Acute Muscle Fatigue Induced by Acute Exercise: a Systematic Review and Meta-analysis
}

\author{
Seunghyeok Yeom \\ Incheon National University \\ Hyungwoo Lee \\ Incheon National University \\ Kyoungkyu Jeon ( $\square$ jeonkay@inu.ac.kr) \\ Incheon National University
}

\section{Research Article}

Keywords: Tensiomyography (TMG), Muscle fatigue, Acute exercise, Meta-analysis

Posted Date: August 23rd, 2021

DOI: https://doi.org/10.21203/rs.3.rs-822118/v1

License: @) (i) This work is licensed under a Creative Commons Attribution 4.0 International License. Read Full License

Version of Record: A version of this preprint was published at Korean Journal of Sport Science on March 31st, 2022. See the published version at https://doi.org/10.24985/kjss.2022.33.1.19. 


\section{Abstract}

Tensiomyography (TMG) has advantage in measuring fatigue. However, no studies have used a meta-analysis approach to analyze the trend of changes in TMG variables for acute muscle fatigue induced by an acute exercise. This study is the first systematic review and meta-analysis to establish criteria for measuring acute fatigue using TMG by quantifying trend of changes in TMG variables. Searches were conducted in Web of Science and Pubmed from December 6, 2020 to January 7, 2021. 16 studies were included that they used in TMG for measuring acute muscle fatigue that was caused by acute exercises in rectus femoris or biceps femoris. The meta-analysis results indicated that in the biceps femoris, showed a significant ( $p<$ .05) decrease in all TMG variables of the elite athletes. Also, in the overall effects of maximum displacement and mean velocity until 90\% Dm (Vc90) showed significant $(p<.05)$ decreasing trend. In the rectus femoris, showed a significant $(p<.05)$ decreasing trend was found for maximum displacement (Dm) in the average person, while contraction time (Tc) showed a decreasing trend in elite athletes and overall. In conclusion, acute muscle fatigue was induced decreased Dm, Tc, Vc90 in the TMG measurement after an acute exercise. These results TMG could be used as a muscle fatigue indicator and help develop a more proper protocol for testing the response of body to muscle fatigue.

\section{Introduction}

Tensiomyography (TMG) is a mechanomyography method that non-invasively evaluates the mechanical properties of skeletal and neuromuscular muscles. It is used to measure the stiffness of a single muscle and neuromuscular muscle fatigue, especially after an acute exercise 1,2,3. TMG induces mechanical contraction by stimulating the muscle with a single electric stimulation of $0-100 \mathrm{~mA}$ for $1 \mathrm{~ms}$ and then measures the response ${ }^{4}$. TMG evaluates the maximum contraction displacement of the muscle (i.e., maximum displacement [Dm]), the time taken to contract from $10-90 \%$ of the maximum contraction displacement (i.e., the contraction time [Tc]), the time taken from the start of stimulation to $10 \%$ of the maximum contraction displacement (i.e., the delay time [Td]), the time from $50 \%$ of the maximum contraction displacement to $50 \%$ of the descending curve (i.e., the sustain time [Ts]), and the time taken from $90-50 \%$ of the maximum contraction displacement in the descending curve (i.e., the relaxation time $[\mathrm{Tr}])$. Measurement-remeasurement and intra-evaluator reliability indices are assessed as Poor (< $0.5)$, Moderate $(0.5$ to $<0.75)$, Good $(0.75$ to $<0.9)$, and Excellent $(\geq 0.9)$ based on the $95 \%$ confidence interval $(\mathrm{Cl})$ of the intraclass correlation coefficient (ICC). Dm (0.91-0.99) and Tc (0.70-0.98) are the most reliable TMG variables in repeated measurements ${ }^{5,6}$. However, Tc can be affected by the Dm size as Tc represents the time taken to reach $90 \%$ of Dm. Therefore, the normalized response speed $(\mathrm{Vrn})$, radial displacement velocity $(\mathrm{Vrd})$, the mean velocity until 10\% Dm (Vc10), and the mean velocity until $90 \% \mathrm{Dm}(\mathrm{Vc} 90)$ compensate for this when calculating muscle contraction speed ${ }^{2,4,7}$.

Running, jumping, and turning movements common in sports occur in the lower body. During running, the knee flexors and extensors compensate for the gastrocnemius activity decrease due to fatigue to maintain the stride pattern ${ }^{8}$. In jumping motions, the hamstrings and quadriceps are elongated to store elastic energy and play an important role in exerting power 9,10 . As the hamstrings and quadriceps play an essential role in lower body movement, they are widely used in post-exercise fatigue measurements. However, fatigue measurement studies using TMG have also been conducted with the biceps femoris (BF) and rectus femoris $(\mathrm{RF})^{4,11,12}$, but the criteria for measuring the degree of fatigue are ambiguous as these previous studies reported inconsistent results. Some reported decreased Dm due to increased muscle stiffness after the induction of fatigue 3,13,14,15,16, while others reported increased Dm due to decreased muscle stiffness ${ }^{12,17}$. Additionally, BF and RF have different responses after the same movement owing to differences in each muscle's role during the movement, which manifests as different responses $4,13,18$.

Although TMG has a potential advantage in measuring fatigue, but the result interpretation is ambiguous as the reported trend of changes in the literature are inconstant. However, no studies have used a meta-analysis approach to analyze the trend of changes in TMG variables for acute muscle fatigue induced by an acute exercise. This study aimed to establish criteria for measuring fatigue using TMG by quantifying trend of changes in TMG variables. 


\section{Methods}

\section{Literature Review}

We performed a literature search, systematic research identification review, and meta-analysis using specific variables. The literature search focused on studies measuring muscle fatigue with TMG before and after an acute exercise to examine muscle condition changes after an acute exercise. The search engines used were PubMed and Web of Science, starting December 6, 2020, and ending January 7, 2021. The keywords were: (1) "Tensiomyography." The literature inclusion criteria were: studies on TMG measurement before and after an acute exercise, RF or BF measurement studies, and studies on TMG measurement immediately after exercise fatigue. The exclusion criteria included unrelated literature, such as review papers, case studies, and animal studies. One researcher conducted the literature search. In total, 282 papers were initially identified, and 266 were excluded after reading the title and summary; 107 were duplicate studies, 11 were review papers, 32 were case studies, and 116 were unrelated subjects. Finally, 16 papers were included in the meta-analysis (Fig. 1) 2,3,4,11,12,13,14,15,16,17,18,19,20,21,22,23.

\section{Tensiomyography}

TMG was used to evaluate muscle fatigue induced by an acute exercise. The included studies evaluated the BF, RF, vastus lateralis (VL), vastus medialis (VM), semitendinosus (ST), and medial gastrocnemius (GM) muscles and the $\mathrm{Dm}, \mathrm{Tc}, \mathrm{Td}, \mathrm{Ts}, \mathrm{Tr}$, Vrn, Vrd, Vc10 and Vc90 TMG measurement variables. For the analysis, Vrn was converted into Tc using the formula $\operatorname{Vrn}=\frac{0.8}{\mathrm{Tc}} 24 . \mathrm{Vc} 90$ was calculated using $\mathrm{Vc} 90=\frac{\mathrm{Dm} * 0.9}{\mathrm{Tc}+\mathrm{Td}}$ to determine the muscle contraction velocity ${ }^{7}$. All of the studies measured TMG before and immediately after exercise. The BF measurement was taken with the subject prone position on a wedge foam cushion with a $5-15^{\circ}$ flexion in the knee joint. The RF measurement was taken with the subject supine position on a special triangular wedge foam cushion with the knee extension at $140-150^{\circ} 25$. During the measurements, the electric potential was administered with an intensity of $0-100 \mathrm{~mA}$ for $1 \mathrm{~ms}$ and gradually increased until maximal $\mathrm{Dm}$. There was at least a $10 \mathrm{~s}$ break between measurements ${ }^{26}$.

This study analyzed two muscles (BF and RF) and three TMG intervention variables (Dm, Tc, and Vc90). Muscle fatigue was induced by the exercise specified in each study ${ }^{2}$, the most measured muscles, and reliable TMG measurement variables were used for our analysis ${ }^{6}$.

\section{Acute Exercise}

The following an acute exercises were used to induce fatigue: repeated sprint ability (RSA), maximal incremental cycling test (MICT), maximal voluntary isometric contraction (MVIC), weight training, running, swimming, forward-tucked somersaults, and surfing.

\section{Meta-analytic approaches}

All statistical data analyses were performed using Comprehensive Meta-Analysis software (version 3.0, Biostat Inc., USA). The individual and overall effect sizes (ES) were calculated using Hedges' $\mathrm{g}$ with $95 \% \mathrm{Cl}^{27}$. Variable increases were labeled as positive, and decreases were labeled as negative. For example, a positive Hedges' g value for Tc, Dm, and Vc90 indicated an increase in each variable after the exercise. For reliability, the ES of all values were calculated, and values more than twice the standard deviation were excluded through a moderate process ${ }^{28}$. Random-effects meta-analysis models were used, assuming that no common ES appeared in individual studies ${ }^{29}$. ES was evaluated as Very Small $(<0.2)$, Small $(0.2$ to $<0.6)$, Average $(0.6$ to $<1.2)$, Large $(1.2$ to $<2.0)$, and Very Large $(\geq 2.0)^{30}$. All meta-analysis results are shown in figure by forest plot.

The Higgins and Green $I^{2}$ test was used to identify the level of heterogeneity in the literature, and an $\mathrm{I}^{2}$ value of $50 \%$ or higher was considered heterogeneous ${ }^{31}$. The methods used for the literature bias test were: (1) the original funnel plot was compared with a revised funnel plot after applying the trim-and-fill technique ${ }^{32}$, and (2) the Egger's regression test was based on the null 
hypothesis that symmetry exists in the funnel plot. Thus, a P-value for the intercept $(\beta 0)<0.05$ indicated a violation of the symmetry assumption, representing a high publication bias ${ }^{33}$.

Finally, the critical appraisal tool (CAT) scale was used to estimate the methodology quality ${ }^{34}$. The CAT consists of 13 questions with five items on the study validity and reliability, four items only on the study validity, and four items only on the reliability (Table 1).

Table 1

The CAT explanation of 13 questions

\begin{tabular}{|c|c|}
\hline Question & Contents \\
\hline $\begin{array}{l}\text { Question } \\
1\end{array}$ & $\begin{array}{l}\text { If human subjects were used, did the authors give a detailed description of the sample of subjects used to } \\
\text { perform the (index) test? }\end{array}$ \\
\hline $\begin{array}{l}\text { Question } \\
2\end{array}$ & Did the authors clarify the qualification, or competence of the rater(s) who performed the (index) test? \\
\hline $\begin{array}{l}\text { Question } \\
3\end{array}$ & Was the reference standard explained? \\
\hline $\begin{array}{l}\text { Question } \\
4\end{array}$ & If interrater reliability was tested, were raters blinded to the findings of other raters? \\
\hline $\begin{array}{l}\text { Question } \\
5\end{array}$ & If intrarater reliability was tested, were raters blinded to their own prior findings of the test under evaluation? \\
\hline $\begin{array}{l}\text { Question } \\
6\end{array}$ & Was the order of examination varied? \\
\hline $\begin{array}{l}\text { Question } \\
7\end{array}$ & $\begin{array}{l}\text { If human subjects were used, was the time period between the reference standard and the index test short } \\
\text { enough to be reasonably sure that the target condition did not change between the two tests? }\end{array}$ \\
\hline $\begin{array}{l}\text { Question } \\
8\end{array}$ & $\begin{array}{l}\text { Was the stability (or theoretical stability) of the variable being measured taken into account when determining } \\
\text { the suitability of the time interval between repeated measures? }\end{array}$ \\
\hline $\begin{array}{l}\text { Question } \\
9\end{array}$ & Was the reference standard independent of the index test? \\
\hline $\begin{array}{l}\text { Question } \\
10\end{array}$ & Was the execution of the (index) test described in sufficient detail to permit replication of the test? \\
\hline $\begin{array}{l}\text { Question } \\
11\end{array}$ & Was the execution of the reference standard described in sufficient detail to permit its replication? \\
\hline $\begin{array}{l}\text { Question } \\
12\end{array}$ & Were withdrawals from the study explained? \\
\hline $\begin{array}{l}\text { Question } \\
13\end{array}$ & Were the statistical methods appropriate for the purpose of the study? \\
\hline
\end{tabular}

The answers to the questions are either yes, no, or not applicable, with 1 point for yes and 0 points for no. As CAT does not provide an overall quality score, the evaluation method by was modified so that the methodological quality could be evaluated 35. A score of 8 points or higher indicated a low risk of bias and excellent methodological quality, 7 points indicated a moderate risk of bias and average methodological quality, and 6 or fewer points indicated a high risk of bias and low methodological quality. Three researchers conducted independent assessments for quality assessment and derived the final score after a discussion.

\section{Results}

\section{Subjects and Study Characteristics}


16 papers met the meta-analysis inclusion criteria and analyzed TMG for muscle fatigue after an acute exercise in 393 healthy participants. 8 papers studied elite athletes, 7 studied amateurs and active individuals, and 1 studied both amateur and elite athletes. Regarding the study design, 9 were single-group pre-posttest studies, 6 were crossover studies, and 1 was a randomized controlled trial (Table 2). 
Table 2

Information of the study

\begin{tabular}{|c|c|c|c|c|c|c|c|c|}
\hline $\begin{array}{l}\text { Study } \\
\text { (Year of } \\
\text { Publication) }\end{array}$ & $\begin{array}{l}\text { Study } \\
\text { Design }\end{array}$ & Participants & $\begin{array}{l}\text { Age } \\
\text { (Year } \\
\pm \text { SD) }\end{array}$ & Gender & $\begin{array}{l}\text { Training } \\
\text { Level }\end{array}$ & Exercise Protocol & $\begin{array}{l}\text { Measure } \\
\text { Muscle }\end{array}$ & $\begin{array}{l}\text { TMG } \\
\text { Variables }\end{array}$ \\
\hline $\begin{array}{l}\text { Barcala- } \\
\text { Furelos } \\
(2020)\end{array}$ & $\begin{array}{l}\text { single } \\
\text { group pre- } \\
\text { posttest }\end{array}$ & 20 & $\begin{array}{l}24.0 \\
\pm 4.9\end{array}$ & $\begin{array}{l}\text { 16Male } \\
\text { 4Female }\end{array}$ & active & $\begin{array}{l}\text { swimming / } 100 \\
\text { m with fins to the } \\
\text { victim, and } \\
\text { towing the victim } \\
100 \mathrm{~m} \text { back } \\
\text { (victim: height, } \\
160-190 \mathrm{~cm} ; \\
\text { weight, } 60-90 \\
\mathrm{~kg} \text { ) }\end{array}$ & RF & $\mathrm{Dm}, \mathrm{Tc}$ \\
\hline $\begin{array}{l}\text { Beato } \\
(2019)\end{array}$ & $\begin{array}{l}\text { randomized } \\
\text { controlled } \\
\text { trial }\end{array}$ & 32 & $\begin{array}{l}21 \pm \\
3\end{array}$ & Male & amateur & $\begin{array}{l}\text { train / } 15 \text { min } \\
\text { workout } \\
\text { programs }\end{array}$ & $\begin{array}{l}\text { VL, VM, } \\
\text { RF }\end{array}$ & $\begin{array}{l}\text { Dm, Tc, } \\
\text { Td, Vc10, } \\
\text { Vc90 }\end{array}$ \\
\hline $\begin{array}{l}\text { Berzosa } \\
(2020)\end{array}$ & Crossover & 10 & $\begin{array}{l}27 \pm \\
1.5\end{array}$ & Male & active & $\begin{array}{l}\text { squat / each } \\
\text { exercise session } \\
\text { consisted of } 4 \\
\text { sets of } 7 \\
\text { repetitions } \\
\text { interspersed by } 2 \\
\text { min between-set } \\
\text { rest intervals. }\end{array}$ & RF, BF & $\mathrm{Dm}, \mathrm{Tc}$ \\
\hline $\begin{array}{l}\text { Calderón- } \\
\text { Pellegrino } \\
(2020)\end{array}$ & $\begin{array}{l}\text { single } \\
\text { group pre- } \\
\text { posttest }\end{array}$ & 32 & $\begin{array}{l}23 \pm \\
5\end{array}$ & Male & elite & $\begin{array}{l}\text { RSA / seven } \\
\text { repeated sprints } \\
\text { of } 30 \mathrm{~m} \text {, with } 20 \mathrm{~s} \\
\text { of active recovery } \\
\text { between }\end{array}$ & RF, BF & $\begin{array}{l}\text { Dm, Tc, } \\
\text { Td }\end{array}$ \\
\hline $\begin{array}{l}\text { García- } \\
\text { García } \\
(2020)\end{array}$ & $\begin{array}{l}\text { single } \\
\text { group pre- } \\
\text { posttest }\end{array}$ & 48 & $\begin{array}{l}20.2 \\
\pm 2.3\end{array}$ & Male & elite & $\begin{array}{l}\text { maximal } \\
\text { incremental } \\
\text { cycling test / } \\
\text { increased } 20 \\
W \cdot \mathrm{min}^{-1} \\
\text { frequency of } 80- \\
90 \mathrm{rev} \cdot \mathrm{min}^{-1} \\
\text { reached an } \\
\text { average of } 393.8 \\
\pm 41.6 \mathrm{~W}(5.8 \pm \\
\left.0.4 \text { Wpeak } \mathrm{kg}^{-1}\right) \\
\text { test duration was } \\
1228 \pm 120 \mathrm{~s}\end{array}$ & $\begin{array}{l}\text { VL, RF, } \\
\text { BF }\end{array}$ & $\begin{array}{l}\text { Dm, Tc, } \\
\text { Td, Ts, } \\
\text { Vrd }\end{array}$ \\
\hline $\begin{array}{l}\text { García- } \\
\text { Unanue } \\
(2020)\end{array}$ & $\begin{array}{l}\text { single } \\
\text { group pre- } \\
\text { posttest }\end{array}$ & 33 & $\begin{array}{l}23.4 \\
\pm 4.4\end{array}$ & Male & $\begin{array}{l}\text { elite / } \\
\text { amateur }\end{array}$ & $\begin{array}{l}\text { RSA / seven } \\
\text { repeated sprints } \\
\text { of } 30 \mathrm{~m} \text {, with } 20 \mathrm{~s} \\
\text { of active recovery } \\
\text { between }\end{array}$ & $\mathrm{RF}, \mathrm{BF}$ & $\begin{array}{l}\text { Dm, Tc, } \\
\text { Td, Ts, Tr }\end{array}$ \\
\hline $\begin{array}{l}\text { López- } \\
\text { Fernández } \\
\text { (2017) }\end{array}$ & Crossover & 16 & $\begin{array}{l}22.2 \\
\pm 3.4\end{array}$ & Male & amateur & $\begin{array}{l}\text { soccer simulation } \\
\text { protocol / } 3 \text { bouts } \\
\text { of the SSP, Each } \\
\text { bout last } 16 \text { min } \\
\text { with } 3 \text { min of rest } \\
\text { between bouts. }\end{array}$ & RF, BF & $\begin{array}{l}\text { Dm, Tc, } \\
\text { Td, Ts, Tr }\end{array}$ \\
\hline
\end{tabular}




\begin{tabular}{|c|c|c|c|c|c|c|c|c|}
\hline $\begin{array}{l}\text { Study } \\
\text { (Year of } \\
\text { Publication) }\end{array}$ & $\begin{array}{l}\text { Study } \\
\text { Design }\end{array}$ & Participants & $\begin{array}{l}\text { Age } \\
\text { (Year } \\
\pm \text { SD) }\end{array}$ & Gender & $\begin{array}{l}\text { Training } \\
\text { Level }\end{array}$ & Exercise Protocol & $\begin{array}{l}\text { Measure } \\
\text { Muscle }\end{array}$ & $\begin{array}{l}\text { TMG } \\
\text { Variables }\end{array}$ \\
\hline $\begin{array}{l}\text { Martín-San } \\
\text { Agustín } \\
(2020)\end{array}$ & $\begin{array}{l}\text { single } \\
\text { group pre- } \\
\text { posttest }\end{array}$ & 35 & $\begin{array}{l}22 \pm \\
2\end{array}$ & $\begin{array}{l}\text { 16Male } \\
\text { 19Female }\end{array}$ & active & $\begin{array}{l}\mathrm{MVIC} / \text { measure } \\
3 \mathrm{MVIC} \text {, and } 60 \mathrm{~s} \\
\text { fatiguing } \\
\text { isometric } \\
\text { contraction at } \\
70 \% \mathrm{MVC}\end{array}$ & $\begin{array}{l}\mathrm{VL}, \mathrm{VM}, \\
\mathrm{RF}\end{array}$ & $\begin{array}{l}\text { Dm, Tc, } \\
\text { Vc10, } \\
\text { Vc90 }\end{array}$ \\
\hline $\begin{array}{l}\text { Pereira } \\
(2020)\end{array}$ & Crossover & 14 & $\begin{array}{l}21.8 \\
\pm 2.6\end{array}$ & Male & elite & $\begin{array}{l}\text { train / } 40-50 \mathrm{~min} \\
\text { workout } \\
\text { programs }\end{array}$ & $\mathrm{RF}, \mathrm{BF}$ & Vc90 \\
\hline $\begin{array}{l}\text { Rodríguez- } \\
\text { Matoso } \\
(2015)\end{array}$ & $\begin{array}{l}\text { single } \\
\text { group pre- } \\
\text { posttest }\end{array}$ & 11 & $\begin{array}{l}28.2 \\
\pm 2.9\end{array}$ & Male & elite & $\begin{array}{l}\text { surfing / wave } \\
\text { size } 1-11 \frac{1}{2} \mathrm{~m} \\
\text { wave length } 6-9 \\
\text { s wave direction } \\
\text { right distance } \\
\text { paddling } 40-50 \\
\mathrm{~m} \text {, heat length } 20 \\
\text { min }\end{array}$ & $\begin{array}{l}\text { VL, VM, } \\
\text { RF, BF, } \\
\text { ST }\end{array}$ & $\begin{array}{l}\text { Dm, Ts, } \\
\text { Tr, Vrn }\end{array}$ \\
\hline $\begin{array}{l}\text { Rojas- } \\
\text { Barrionuevo } \\
(2017)\end{array}$ & Crossover & 14 & $\begin{array}{l}20.7 \\
\pm 3.1\end{array}$ & Male & elite & $\begin{array}{l}\text { forward tucked } \\
\text { somersaults / } 12 \\
\text { sets of } 6 \\
\text { repetitions of } \\
\text { forward tucked } \\
\text { somersaults, a } \\
\text { rest period of } 2 \\
\text { min between sets, } \\
5 \text { s between } \\
\text { repetitions was } \\
\text { implemented }\end{array}$ & $\begin{array}{l}\mathrm{VL}, \mathrm{VM}, \\
\mathrm{RF}, \mathrm{BF} \\
\mathrm{GM}\end{array}$ & Vrn \\
\hline $\begin{array}{l}\text { Rojas- } \\
\text { Valverde } \\
(2020)\end{array}$ & Crossover & 20 & $\begin{array}{l}20.4 \\
\pm 3.2\end{array}$ & Male & active & $\begin{array}{l}\text { running / } 30 \mathrm{~min} \\
\text { running }\end{array}$ & $\mathrm{RF}, \mathrm{BF}$ & $\mathrm{Dm}, \mathrm{Tc}$ \\
\hline $\begin{array}{l}\text { Sánchez- } \\
\text { Sánchez } \\
\text { (2018) }\end{array}$ & $\begin{array}{l}\text { single } \\
\text { group pre- } \\
\text { posttest }\end{array}$ & 20 & $\begin{array}{l}25.5 \\
\pm 6.1\end{array}$ & Male & elite & $\begin{array}{l}\text { RSA / seven } \\
\text { repeated sprints } \\
\text { of } 30 \mathrm{~m} \text {, with } 20 \mathrm{~s} \\
\text { of active recovery } \\
\text { between }\end{array}$ & $\mathrm{RF}, \mathrm{BF}$ & $\begin{array}{l}\text { Dm, Tc, } \\
\text { Td, Ts }\end{array}$ \\
\hline $\begin{array}{l}\text { Sánchez- } \\
\text { Sánchez } \\
\text { (2019) }\end{array}$ & $\begin{array}{l}\text { single } \\
\text { group pre- } \\
\text { posttest }\end{array}$ & 62 & $\begin{array}{l}14.6 \\
\pm 2.0\end{array}$ & $\begin{array}{l}\text { Male } \\
\text { Female }\end{array}$ & elite & $\begin{array}{l}\text { RSA / seven } \\
\text { repeated sprints } \\
\text { of } 30 \mathrm{~m} \text {, with } 20 \mathrm{~s} \\
\text { of active recovery } \\
\text { between }\end{array}$ & $\mathrm{RF}, \mathrm{BF}$ & $\begin{array}{l}\text { Dm, Tc, } \\
\text { Td }\end{array}$ \\
\hline $\begin{array}{l}\text { Santana } \\
(2018)\end{array}$ & $\begin{array}{l}\text { single } \\
\text { group pre- } \\
\text { posttest }\end{array}$ & 11 & $\begin{array}{l}20.8 \\
\pm 3.2\end{array}$ & Male & elite & $\begin{array}{l}\text { forward tucked } \\
\text { somersaults / } 12 \\
\text { sets of } 6 \\
\text { repetitions of } \\
\text { forward tucked } \\
\text { somersaults, A } \\
\text { rest period of } 2 \\
\text { min between sets, } \\
5 \text { s between } \\
\text { repetitions was } \\
\text { implemented }\end{array}$ & $\begin{array}{l}\text { VL, VM, } \\
\mathrm{RF}, \mathrm{BF} \\
\mathrm{GM}\end{array}$ & $\begin{array}{l}\text { Dm, Tc, } \\
\text { Td, Vrn }\end{array}$ \\
\hline
\end{tabular}




\begin{tabular}{|c|c|c|c|c|c|c|c|c|}
\hline $\begin{array}{l}\text { Study } \\
\text { (Year of } \\
\text { Publication) }\end{array}$ & $\begin{array}{l}\text { Study } \\
\text { Design }\end{array}$ & Participants & $\begin{array}{l}\text { Age } \\
\text { (Year } \\
\pm \text { SD) }\end{array}$ & Gender & $\begin{array}{l}\text { Training } \\
\text { Level }\end{array}$ & Exercise Protocol & $\begin{array}{l}\text { Measure } \\
\text { Muscle }\end{array}$ & $\begin{array}{l}\text { TMG } \\
\text { Variables }\end{array}$ \\
\hline $\begin{array}{l}\text { Ubago } \\
\text { Guisado } \\
(2017)\end{array}$ & Crossover & 15 & $\begin{array}{l}23.4 \\
\pm 3.4\end{array}$ & Female & amateur & $\begin{array}{l}\text { modified RSA / } \\
\text { consisted of six } \\
\text { sprints of } 40 \text { m } \\
(20+20 \text { m) with } \\
20 \text { s of passive } \\
\text { recovery }\end{array}$ & $\mathrm{RF}, \mathrm{BF}$ & $\begin{array}{l}\text { Dm, Tc, } \\
\text { Td, Tr }\end{array}$ \\
\hline
\end{tabular}

Caption RSA repeated sprint ability, MVIC maximal voluntary isometric contraction BF biceps femoris, $R F$ rectus femoris, VL vastus lateralis, VM vastus medialis, ST semitendinosus, GM medial gastrocnemius Dm maximum displacement, TC contraction time, Td delay time, Ts sustain time, Tr relaxation time, Vrd radial displacement velocity, Vrn normalized response speed, Vc10 mean velocity until $10 \% \mathrm{Dm}$, Vc90 mean velocity until $90 \% \mathrm{Dm}$

\section{Tensiomyography}

All 16 studies analyzed RF, 13 studies measured BF, 6 measured VL, 5 measured VM, 1 measured ST, and 2 measured GM. Regarding TMG variables, 14 studies measured Dm, 13 measured Tc, 9 measured Td, 3 measured Vc90, 1 measured Vc10, 5 measured Ts, 4 measured Tr, 3 measured Vrn, and 1 measured Vrd. Papers that presented Dm, Tc, and Td together used the Vc90 calculation formula to convert them into Vc90 (Table 2).

\section{Exercise Protocols}

Of the 16 studies, 4 used RSA, 1 used MICT, 1 used MVIC, 3 used weight training, 3 used running, 1 used swimming, 2 used forward-tucked somersaults, and 1 used surfing to induce acute muscle fatigue (Table 2).

\section{Methodological Bias Assessment}

The mean CAT score and SD was $9.5 \pm 1.5$ (range 6-11), 14 papers had a low risk of bias and good methodological quality, 1 had a moderate risk of bias and average methodological quality, and 1 had a high risk of bias and low methodological quality (Table 3). 
Table 3

Quility and risk of bias assessment of the included validity studies, utilized with the critical appraisal tool (CAT)

\begin{tabular}{|c|c|c|c|c|c|c|c|c|c|c|c|c|c|c|c|}
\hline $\begin{array}{l}\text { Author } \\
\text { (Year of } \\
\text { Publication) }\end{array}$ & Q1 & Q2 & Q3 & Q4 & Q5 & Q6 & Q7 & Q8 & Q9 & Q10 & Q11 & Q12 & Q13 & Total & $\begin{array}{l}\text { Risk of } \\
\text { bias }\end{array}$ \\
\hline $\begin{array}{l}\text { Barcala- } \\
\text { Furelos } \\
\text { (2020) }\end{array}$ & $\mathrm{Y}$ & Y & $Y$ & N/A & $\mathrm{N} / \mathrm{A}$ & $\mathrm{N}$ & $\mathrm{Y}$ & $\mathrm{N}$ & $\mathrm{N}$ & $Y$ & Y & $\mathrm{Y}$ & $Y$ & $8 / 11$ & Low \\
\hline $\begin{array}{l}\text { Beato } \\
(2019)\end{array}$ & Y & $N$ & Y & N/A & $\mathrm{N} / \mathrm{A}$ & Y & $N$ & $Y$ & Y & $Y$ & Y & Y & Y & $9 / 11$ & Low \\
\hline $\begin{array}{l}\text { Berzosa } \\
(2020)\end{array}$ & $\mathrm{Y}$ & $N$ & Y & N/A & $\mathrm{N} / \mathrm{A}$ & $\mathrm{N}$ & Y & $\mathrm{N}$ & $\mathrm{N}$ & $Y$ & Y & Y & Y & $7 / 11$ & Moderate \\
\hline $\begin{array}{l}\text { Calderon- } \\
\text { Pellegrino } \\
(2020)\end{array}$ & Y & Y & Y & N/A & $\mathrm{N} / \mathrm{A}$ & $\mathrm{Y}$ & $\mathrm{N}$ & Y & Y & $Y$ & Y & Y & Y & $10 / 11$ & Low \\
\hline $\begin{array}{l}\text { Garcia- } \\
\text { Garcia } \\
(2020)\end{array}$ & Y & $N$ & $Y$ & N/A & $\mathrm{N} / \mathrm{A}$ & Y & $N$ & Y & $\mathrm{Y}$ & $Y$ & Y & Y & Y & $9 / 11$ & Low \\
\hline $\begin{array}{l}\text { Garcia- } \\
\text { Unanue } \\
\text { (2020) }\end{array}$ & $\mathrm{Y}$ & $\mathrm{Y}$ & Y & $\mathrm{N} / \mathrm{A}$ & $\mathrm{N} / \mathrm{A}$ & $\mathrm{Y}$ & $\mathrm{N}$ & $\mathrm{Y}$ & $\mathrm{Y}$ & $Y$ & Y & Y & Y & $10 / 11$ & Low \\
\hline $\begin{array}{l}\text { Lopez- } \\
\text { Fernandez } \\
\text { (2017) }\end{array}$ & Y & $Y$ & Y & N/A & $\mathrm{N} / \mathrm{A}$ & Y & $Y$ & $\mathrm{Y}$ & $Y$ & Y & Y & Y & Y & $11 / 11$ & Low \\
\hline $\begin{array}{l}\text { Martin-San } \\
\text { Agustin } \\
(2020)\end{array}$ & Y & $Y$ & Y & N/A & $\mathrm{N} / \mathrm{A}$ & $\mathrm{Y}$ & $\mathrm{Y}$ & Y & $Y$ & $Y$ & Y & Y & Y & $11 / 11$ & Low \\
\hline $\begin{array}{l}\text { Pereira } \\
(2020)\end{array}$ & Y & Y & Y & N/A & $\mathrm{N} / \mathrm{A}$ & Y & Y & Y & Y & $Y$ & Y & Y & Y & $11 / 11$ & Low \\
\hline $\begin{array}{l}\text { Rodriguez- } \\
\text { Matoso } \\
\text { (2015) }\end{array}$ & $\mathrm{Y}$ & $N$ & $N$ & N/A & $\mathrm{N} / \mathrm{A}$ & $\mathrm{Y}$ & $\mathrm{N}$ & Y & $Y$ & $\mathrm{~N}$ & $N$ & $\mathrm{Y}$ & $\mathrm{Y}$ & $6 / 11$ & High \\
\hline $\begin{array}{l}\text { Rojas- } \\
\text { Barrionuevo } \\
\text { (2017) }\end{array}$ & $Y$ & $Y$ & $\mathrm{Y}$ & N/A & $\mathrm{N} / \mathrm{A}$ & $Y$ & Y & $N$ & $Y$ & $Y$ & $Y$ & $Y$ & $Y$ & $10 / 11$ & Low \\
\hline $\begin{array}{l}\text { Rojas- } \\
\text { Valverde } \\
\text { (2020) }\end{array}$ & $Y$ & $N$ & $Y$ & N/A & $\mathrm{N} / \mathrm{A}$ & $Y$ & $Y$ & $Y$ & $Y$ & $Y$ & $Y$ & $Y$ & $Y$ & $10 / 11$ & Low \\
\hline $\begin{array}{l}\text { Sanchez- } \\
\text { Sanchez } \\
(2018)\end{array}$ & $\mathrm{Y}$ & $Y$ & $Y$ & N/A & $\mathrm{N} / \mathrm{A}$ & $Y$ & $\mathrm{~N}$ & $Y$ & $Y$ & $Y$ & $Y$ & $Y$ & $Y$ & $10 / 11$ & Low \\
\hline $\begin{array}{l}\text { Sanchez- } \\
\text { Sanchez } \\
(2019)\end{array}$ & $Y$ & $Y$ & $Y$ & $\mathrm{~N} / \mathrm{A}$ & $\mathrm{N} / \mathrm{A}$ & $Y$ & $Y$ & $Y$ & $Y$ & $Y$ & $Y$ & $Y$ & $Y$ & $11 / 11$ & Low \\
\hline $\begin{array}{l}\text { Santana } \\
\text { (2018) }\end{array}$ & $Y$ & $\mathrm{~N}$ & $Y$ & N/A & $\mathrm{N} / \mathrm{A}$ & $\mathrm{Y}$ & $Y$ & $Y$ & $Y$ & $Y$ & $Y$ & $Y$ & $Y$ & $10 / 11$ & Low \\
\hline $\begin{array}{l}\text { Ubago } \\
\text { (2017) }\end{array}$ & $Y$ & $Y$ & $Y$ & N/A & $\mathrm{N} / \mathrm{A}$ & $Y$ & $N$ & $N$ & $Y$ & $Y$ & $Y$ & Y & Y & $9 / 11$ & Low \\
\hline
\end{tabular}

Caption Q question, Y yes, N no, N/A not applicable 


\section{Heterogeneity Evaluation and publication bias}

The overall heterogeneity was $\mathrm{I}^{2}=35.77 \% \pm 30.30(\mathrm{SD})$. It was either moderate or high. Some studies had significant Q-value, in the RF Dm that showed elite athletes $\mathrm{I}^{2}: 55.47 \%$ and overall $\mathrm{I}^{2}=52.67 \%$. The BF Vc90 showed elite athletes $\mathrm{I}^{2}=60.71 \%$ and overall $I^{2}=55.15 \%$. The RF Vc90 showed an average person $I^{2}=85.01 \%$, elite athletes $I^{2}=80.11 \%$, and overall $I^{2}=82.28 \%$. However, there $\mathrm{I}^{2}$ were insignificantly heterogeneous. And all analysis were not publication bias

\section{Comparison Between Elite Athletes and Average Persons}

The random effects meta-analysis model analyzed the differences in the TMG variables that measured BF and RF muscle fatigue after an acute exercise.

The ES of BF Dm was insignificant for the average person (Hedges's $\mathrm{g}=0.007,95 \% \mathrm{Cl}=-0.189$ to $0.202, \mathrm{p}=0.946, \mathrm{I}^{2}=0.00 \%$ ), whereas the elite athletes showed a small but significant decrease (Hedges's $g=-0.386,95 \% \mathrm{Cl}=-0.566$ to $-0.207, p=0.000, \mathrm{I}^{2}$ $=9.50 \%$ ). The overall effect also showed a small but significant decrease (Hedges's $\mathrm{g}=-0.207,95 \% \mathrm{Cl}=-0.339$ to $-0.075, \mathrm{p}=$ $0.002, I^{2}=24.51 \%$ ). And the ES of RF Dm showed a small but significant decrease for the average person (Hedges's $g=-0.298$, $95 \% \mathrm{Cl}=-0.518$ to $-0.078, p=0.008, I^{2}=34.94 \%$ ), whereas the change was insignificant in elite athletes (Hedges's $g=0.132$, $95 \% \mathrm{Cl}=-0.101$ to $0.366, \mathrm{p}=0.266, \mathrm{I}^{2}=55.47 \%$ ). The overall effect was statistically insignificant (Hedges's $\mathrm{g}=-0.095,95 \% \mathrm{Cl}=$ -0.256 to $0.065, p=0.242,1^{2}=52.67 \%$ ) (Fig. 2).

The ES of BF Tc change was insignificant in the average person (Hedges's $\mathrm{g}=0.014,95 \% \mathrm{Cl}=-0.180$ to $0.209, \mathrm{p}=0.885, \mathrm{I}^{2}=$ $0.00 \%$ ), but showed a very small but significant decrease in elite athletes (Hedges's $g=-0.164,95 \% \mathrm{Cl}=-0.316$ to $-0.013, p=$ $0.033, I^{2}=0.00 \%$ ). However, the overall effect was statistically insignificant (Hedges's $\mathrm{g}=-0.097,95 \% \mathrm{Cl}=-0.216$ to $0.022, \mathrm{p}=$ $0.111, \mathrm{I}^{2}=0.00 \%$ ). And the ES of RF Tc change was insignificant in the average person (Hedges's $\mathrm{g}=-0.144,95 \% \mathrm{Cl}=-0.304$ to $0.016, p=0.078, I^{2}=0.00 \%$ ), yet showed a very small but significant decrease in elite athlete (Hedges's $\mathrm{g}=-0.172,95 \% \mathrm{Cl}=$ -0.336 to $\left.-0.007, p=0.041, I^{2}=8.51 \%\right)$. The overall effect also showed a very small but significant decrease (Hedges's $g=$ $-0.157,95 \% \mathrm{Cl}=-0.272$ to $-0.043, \mathrm{p}=0.007, \mathrm{I}^{2}=0.00 \%$ ) (Fig. 3).

The ES of BF Vc90 change was insignificant in the average person (Hedges's $\mathrm{g}=-0.159,95 \% \mathrm{Cl}=-0.498$ to $0.181, \mathrm{p}=0.359, \mathrm{I}^{2}$ $=15.20 \%$ ), but showed a small but significant decrease in elite athletes (Hedges's $\mathrm{g}=-0.387,95 \% \mathrm{Cl}=-0.663$ to $-0.111, \mathrm{p}=$ $0.006, \mathrm{I}^{2}=60.71 \%$ ). The overall effect also showed a small but significant decrease (Hedges's $\mathrm{g}=-0.296,95 \% \mathrm{Cl}=-0.510$ to $-0.082, p=0.007, I^{2}=55.15 \%$ ). And the ES of RF Vc90 change was insignificant for the average person (Hedges's $g=0.335,95 \%$ $\mathrm{Cl}=-0.295$ to $0.965, \mathrm{p}=0.298, \mathrm{I}^{2}=85.01 \%$ ), elite athletes (Hedges's $\mathrm{g}=0.115,95 \% \mathrm{Cl}=-0.253$ to $0.484, p=0.540, \mathrm{I}^{2}=80.11 \%$ ), and in the overall effect (Hedges's $\mathrm{g}=0.171,95 \% \mathrm{Cl}=-0.147$ to $0.489, \mathrm{p}=0.292, \mathrm{I}^{2}=82.28 \%$ ) (Fig. 4).

Thus, in BF a substantial decreasing trend in all variables was observed in the elite athletes, with a substantial decreasing trend in the overall effects of Dm and Vc90. Whereas, in RF a substantial decreasing trend was found for Tc in the elite athletes, while Dm was substantial decreasing trend in the average person.

\section{Discussion}

This study is the first to conduct a systematic literature review and meta-analysis on TMG variable trend of changes when measuring acute muscle fatigue induced by an acute exercise using a large sample number obtained from the literature. The CAT evaluation showed that the bias risk and the methodological level were excellent. Further, the meta-analysis results confirmed a specific trend of changes when measuring acute muscle fatigue induced by an acute exercise with TMG, and the trend of changes were different for BF and RF.

The literature analysis demonstrated a substantial decrease in the overall effect in BF Dm and Vc90 and RF Tc. The ES of BF Dm was Hedges's $g=-0.207$, the ES of BF Vc90 was Hedges's $g=-0.296$, and the ES of RF Tc was Hedges's $g=-0.157$. Elite athletes showed a substantial decrease in all BF variables and RF Tc after an acute exercise. The ES was Hedges's $g=-0.386$ 
for BF Dm, Hedges's $g=-0.164$ for BF Tc, Hedges's $g=-0.387$ for BF Vc90, and Hedges's $g=-0.172$ for RF Tc. Conversely, in the average person only the RF Dm showed a substantial decrease, and the ES of RF Dm was Hedges's $g=-0.298$.

Regarding the overall ES, there was a substantial decrease in the BF Vc90, which is consistent with the results of a study that reported a decrease in $\mathrm{Vc} 90$ after an acute exercise ${ }^{2,22}$. This result is owing to the $\mathrm{pH}$ inside the muscle fibers, which decreases due to acute muscle fatigue, reducing the transmission rate of the action potential and affecting the contraction force and contraction rate. A decrease in the number of adenosine triphosphates may cause a decrease in the contractile force by reducing actin-myosin cross-bridge. Additionally, the ratio of fast-twitch muscle fibers (Type II) in the quadriceps and hamstrings is higher than slow-twitch muscle fibers (Type I). Type II muscle fibers have fewer mitochondria and less oxidative metabolism for energy production than Type I, resulting in low fatigue resistance, potentially leading to a contraction rate decrease by becoming fatigued first during exercise $36,37,38,39,40$. Furthermore, there was a substantial decreasing trend of changes in Dm, which is consistent with previous results reporting that muscle fatigue after an exercise increases tension and stiffness $3,13,15,16,21$.

That study reported that muscle fatigue causes a decrease in $\mathrm{pH}$ from accumulating $\mathrm{H}^{+}$and $\mathrm{Pi}$ in the muscle fiber, thereby increasing $\mathrm{Ca}^{2+}$, which reduces the sensitivity of troponin to $\mathrm{Ca}^{2+}$ and inhibits reabsorption in the sarcoplasmic reticulum. Ultimately, stiffness increases as a result of a reduced actin-myosin bond separation rate ${ }^{38,40}$. This phenomenon can be thought of as a way to store more energy generated during exercise by increasing muscle stiffness instead of decreasing muscle activity when muscle fatigue increases ${ }^{41}$.

RF only showed a substantial decreasing trend for Tc, likely explained by the fact that the acute exercise used in each study did not cause substantial muscle fatigue. Several studies included in the analysis reported that the exercise was not enough to induce fatigue in $\mathrm{RF}^{20,23}$, and reported an improvement in the movement response time due to the post-exercise post-activation potentiation (PAP), which can induce a Tc reduction. Additionally, although not substantial, the RF Vc90 slightly increased, which was affected by the inclusion of some studies that reported such results ${ }^{22}$.

There was a difference in the fatigue measurement results between the average person and elite athletes. The average person only showed a substantial decrease in the RF Dm, whereas elite athletes showed a substantial decrease in the all BF variables and RF Tc. This difference is thought to be because the acute exercise used in the literature had many movements requiring explosive muscle strength where hamstring activity is very important, such as running and jumping ${ }^{42}$. The degree of fatigue could have also been affected by the difference in the level of muscular activation during exercise, as there are differences between an average person and an elite athlete in their performance ability and training level, as demonstrated by previous studies $43,44,45$. Previous studies detailed that a higher training level shows a faster and stronger expression of muscular strength and greater activity $46,47,48$. Therefore, the athletes likely generated a greater force in the hamstrings during the exercise compared to the average person, perhaps resulting in a higher fatigue level after exercise ${ }^{46,47,49}$. The quadriceps assist with knee stability during exercise, and during explosive movements, it creates motion in coordination with the hip extensor muscle $50,51,52$. In the acute exercise used in these studies, RF performs this role and is thought to explain the decrease in Tc and Dm in elite athletes and average persons.

This analysis had some limitations. The number of papers was small because research using TMG is relatively new. Further, the muscle fatigue response induced by acute exercise was reported differently in each study ${ }^{3,53}$. Therefore, to identify trend of changes, more studies measuring acute muscle fatigue induced by acute exercise are required. Furthermore, the exercises used in the studies are multiple joint exercise, which could have been inappropriate for inducing localized fatigue ${ }^{54,55}$. Thus, it is necessary to use an exercise that induces fatigue exactly at the measurement site ${ }^{52,56,57}$, and additional research should verify whether the exercise, the amount, and the intensity are sufficient. Further, a study that reported the PAP response due to multiple joint exercise was also included in our analysis. However, PAP wasn't clear how it works so continual research on exercise and PAP response is also necessary ${ }^{58,59}$. Lastly, there was only one researcher who collected papers, and only English publications were considered for this study, potentially causing unintentional sampling bias.

Page $11 / 18$ 


\section{Conclusions}

Our study results indicate that acute muscle fatigue was induced if Dm, Tc, and Vc90 decreased in TMG measurement after an acute exercise. These results could be used as a muscle fatigue indicator when attempting to induce fatigue through exercise, which could help develop a more suitable protocol for testing the body's response to muscle fatigue. Athletes could also check their fatigue level after exercise by checking for decreases in Dm, Tc, and Vc90 in the BF after a complex lower body movement, which could help optimize exercise programs.

\section{Declarations}

\section{Conflict of interest}

The authors report no conflict of interest.

\section{Acknowledgements}

None.

\section{References}

1. Rusu, L. D. et al. Tensiomyography method used for neuromuscular assessment of muscle training. Journal of neuroengineering and rehabilitation 10, 1-8 (2013).

2. Pereira, L. A. et al. Is Tensiomyography-Derived Velocity of Contraction a Sensitive Marker to Detect Acute Performance Changes in Elite Team-Sport Athletes? International Journal of Sports Physiology and Performance 15, 31-37 (2020).

3. García-García, O. et al. A maximal incremental test in cyclists causes greater peripheral fatigue in biceps femoris. Research quarterly for exercise and sport 91, 460-468 (2020).

4. García-Unanue, J. et al. Muscular and physical response to an agility and repeated sprint tests according to the level of competition in futsal players. Frontiers in Psychology 11, 3671 (2020).

5. Križaj, D., Šimunič, B. \& Žagar, T. Short-term repeatability of parameters extracted from radial displacement of muscle belly. Journal of Electromyography and Kinesiology 18, 645-651 (2008).

6. Martín-Rodríguez, S., Loturco, I., Hunter, A. M., Rodríguez-Ruiz, D. \& Munguia-Izquierdo, D. Reliability and measurement error of tensiomyography to assess mechanical muscle function: A systematic review. The Journal of Strength \& Conditioning Research 31, 3524-3536 (2017).

7. de Paula Simola, R. Á. et al. Assessment of neuromuscular function after different strength training protocols using tensiomyography. The Journal of Strength \& Conditioning Research 29, 1339-1348 (2015).

8. Rabita, G., Couturier, A., Dorel, S., Hausswirth, C. \& Le Meur, Y. Changes in spring-mass behavior and muscle activity during an exhaustive run at V02max. Journal of biomechanics 46, 2011-2017 (2013).

9. Peng, H.-T., Kernozek, T. W. \& Song, C.-Y. Quadricep and hamstring activation during drop jumps with changes in drop height. Physical Therapy in Sport 12, 127-132 (2011).

10. Padulo, J. et al. EMG amplitude of the biceps femoris during jumping compared to landing movements. Springerplus 2, 1-7 (2013).

11. Santana, M. V., Rojas, N. A., Montosa, I. \& Bedoya, J. L. Application of tensiomyography to assess the muscle response in the lower limbs of acrobatic gymnasts. European Journal of Human Movement, 96-110 (2018).

12. López-Fernández, J. et al. Neuromuscular responses and physiological patterns during a soccer simulation protocol. Artificial turf versus natural grass. J Sports Med Phys Fitness 22, 07768-07764 (2017).

13. Sánchez-Sánchez, J. et al. Repeated sprint ability and muscular responses according to the age category in elite youth soccer players. Frontiers in physiology 10, 175 (2019). 
14. Martín-San Agustín, R. et al. Tensiomyographical responsiveness to peripheral fatigue in quadriceps femoris. PeerJ 8 , e8674 (2020).

15. Calderón-Pellegrino, G. et al. Influence of artificial turf temperature on physical performance and muscle contractile properties in football players after a repeated-sprint ability test. Scientific Reports 10, 1-8 (2020).

16. Berzosa, C. et al. Effect of Three Half-Squat Protocols on the Tensiomyographic Twitch Response and Tissue Damage of the Rectus Femoris and the Biceps Femoris. Journal of Human Kinetics 75, 15-27 (2020).

17. Rojas-Barrionuevo, N. A., Vernetta-Santana, M., Alvariñas-Villaverde, M. \& López-Bedoya, J. Acute effect of acrobatic jumps on different elastic platforms in the muscle response evaluated through tensiomyography. (2017).

18. Sánchez-Sánchez, J. et al. Effect of a Repeated Sprint Ability test on the muscle contractile properties in elite futsal players. Scientific Reports 8, 1-8 (2018).

19. Ubago Guisado, E. et al. Muscle contractile properties on different sport surfaces using tensiomyography. (2017).

20. Rojas-Valverde, D., Ugalde-Ramírez, J. A., Sánchez-Ureña, B. \& Gutiérrez-Vargas, R. Influence of Altitude and Environmental Temperature on Muscle Functional and Mechanical Activation After 30'Time Trial Run. MHSalud 17, 19-33 (2020).

21. Rodríguez-Matoso, D. et al. Mechanical response of knee muscles in high level bodyboarders during performance. Revista Brasileira de Medicina do Esporte 21, 144-147 (2015).

22. Beato, M., Madruga-Parera, M., Piqueras-Sanchiz, F., Moreno-Pérez, V. \& Romero-Rodriguez, D. Acute Effect of Eccentric Overload Exercises on Change of Direction Performance and Lower-Limb Muscle Contractile Function. Journal of strength and conditioning research (2019).

23. Barcala-Furelos, R. et al. Is Low-Frequency Electrical Stimulation a Tool for Recovery after a Water Rescue? A Cross-Over Study with Lifeguards. International Journal of Environmental Research and Public Health 17, 5854 (2020).

24. Park, S. Theory and usage of tensiomyography and the analysis method for the patient with low back pain. Journal of Exercise Rehabilitation 16, 325 (2020).

25. García-García, O., Cuba-Dorado, A., Fernández-Redondo, D. \& López-Chicharro, J. Neuromuscular parameters predict the performance in an incremental cycling test. International journal of sports medicine 39, 909-915 (2018).

26. Hunter, A. M. et al. Assessment of eccentric exercise-induced muscle damage of the elbow flexors by tensiomyography. Journal of Electromyography and Kinesiology 22, 334-341 (2012).

27. Hedges, L. V. \& Olkin, I. Statistical methods for meta-analysis. (Academic press, 2014).

28. Bodner, T. E. Standardized effect sizes for moderated conditional fixed effects with continuous moderator variables. Frontiers in psychology 8, 562 (2017).

29. Borenstein, M., Hedges, L. V., Higgins, J. P. \& Rothstein, H. R. A basic introduction to fixed-effect and random-effects models for meta-analysis. Research synthesis methods 1, 97-111 (2010).

30. Hopkins, W., Marshall, S., Batterham, A. \& Hanin, J. Progressive statistics for studies in sports medicine and exercise science. Medicine+ Science in Sports+ Exercise 41, 3 (2009).

31. Higgins, J. P. \& Thompson, S. G. Quantifying heterogeneity in a meta-analysis. Statistics in medicine 21, 1539-1558 (2002).

32. Duval, S. \& Tweedie, R. Trim and fill: a simple funnel-plot-based method of testing and adjusting for publication bias in meta-analysis. Biometrics 56, 455-463 (2000).

33. Egger, M., Smith, G. D., Schneider, M. \& Minder, C. Bias in meta-analysis detected by a simple, graphical test. Bmj 315, 629634 (1997).

34. Brink, Y. \& Louw, Q. A. Clinical instruments: reliability and validity critical appraisal. Journal of evaluation in clinical practice 18, 1126-1132 (2012).

35. Carlsson, H. \& Rasmussen-Barr, E. Clinical screening tests for assessing movement control in non-specific low-back pain. A systematic review of intra-and inter-observer reliability studies. Manual therapy 18, 103-110 (2013).

36. Meznaric, M. \& Cvetko, E. Size and proportions of slow-twitch and fast-twitch muscle fibers in human costal diaphragm. BioMed research international 2016 (2016). 
37. Li, B. et al. Profiling and functional analysis of circular RNAs in porcine fast and slow muscles. Frontiers in Cell and Developmental Biology 8, 322 (2020).

38. Fitts, R. H. The cross-bridge cycle and skeletal muscle fatigue. Journal of applied physiology 104, 551-558 (2008).

39. Evangelidis, P. E. et al. The functional significance of hamstrings composition: is it really a "fast" muscle group? Scandinavian journal of medicine \& science in sports 27, 1181-1189 (2017).

40. Boyas, S. \& Guével, A. Neuromuscular fatigue in healthy muscle: underlying factors and adaptation mechanisms. Annals of physical and rehabilitation medicine 54, 88-108 (2011).

41. Dumke, C. L., Pfaffenroth, C. M., McBride, J. M. \& McCauley, G. O. Relationship between muscle strength, power and stiffness and running economy in trained male runners. International journal of sports physiology and performance 5 , 249261 (2010).

42. Morin, J.-B. et al. Sprint acceleration mechanics: the major role of hamstrings in horizontal force production. Frontiers in physiology 6, 404 (2015).

43. Zhang, J. H. et al. How do training experience and geographical origin of a runner affect running biomechanics? Gait \& Posture 84, 209-214 (2021).

44. Maniar, N., Schache, A., Heiderscheit, B. \& Opar, D. in Prevention and Rehabilitation of Hamstring Injuries 65-81 (Springer, 2020).

45. Hébert-Losier, K., Mourot, L. \& Holmberg, H.-C. Elite and amateur orienteers' running biomechanics on three surfaces at three speeds. Medicine \& Science in Sports \& Exercise 47, 381-389 (2015).

46. Schoenfeld, B. J., Contreras, B., Willardson, J. M., Fontana, F. \& Tiryaki-Sonmez, G. Muscle activation during low-versus high-load resistance training in well-trained men. European journal of applied physiology 114, 2491-2497 (2014).

47. Janusevicius, D. et al. Effects of high velocity elastic band versus heavy resistance training on hamstring strength, activation, and sprint running performance. Journal of sports science \& medicine 16, 239 (2017).

48. Higashihara, A., Nagano, Y., Ono, T. \& Fukubayashi, T. Differences in hamstring activation characteristics between the acceleration and maximum-speed phases of sprinting. Journal of sports sciences $\mathbf{3 6}, 1313-1318$ (2018).

49. Chumanov, E. S., Heiderscheit, B. C. \& Thelen, D. G. Hamstring musculotendon dynamics during stance and swing phases of high speed running. Medicine and science in sports and exercise 43, 525 (2011).

50. Shaw, T., Williams, M. T. \& Chipchase, L. S. Do early quadriceps exercises affect the outcome of ACL reconstruction? A randomised controlled trial. Australian Journal of Physiotherapy 51, 9-17 (2005).

51. Lewek, M., Rudolph, K., Axe, M. \& Snyder-Mackler, L. The effect of insufficient quadriceps strength on gait after anterior cruciate ligament reconstruction. Clinical biomechanics 17, 56-63 (2002).

52. Kakehata, G., Goto, Y., Iso, S. \& Kanosue, K. Timing of Rectus Femoris and Biceps Femoris Muscle Activities in Both Legs at Maximal Running Speed. Medicine and Science in Sports and Exercise 53, 643-652 (2021).

53. Piqueras-Sanchiz, F. et al. Effects of moderate vs. high iso-inertial loads on power, velocity, work and hamstring contractile function after flywheel resistance exercise. PLoS One 14, e0211700 (2019).

54. Moore, R. D., Romine, M. W., O'connor, P. J. \& Tomporowski, P. D. The influence of exercise-induced fatigue on cognitive function. Journal of sports sciences 30, 841-850 (2012).

55. Marino, F. E., Gard, M. \& Drinkwater, E. J. The limits to exercise performance and the future of fatigue research. British journal of sports medicine 45, 65-67 (2011).

56. MacKenzie, S. J., Lavers, R. J. \& Wallace, B. B. A biomechanical comparison of the vertical jump, power clean, and jump squat. Journal of sports sciences 32, 1576-1585 (2014).

57. Higashihara, A., Ono, T., Kubota, J., Okuwaki, T. \& Fukubayashi, T. Functional differences in the activity of the hamstring muscles with increasing running speed. Journal of sports sciences 28, 1085-1092 (2010).

58. Batista, M. A. et al. Intermittent exercise as a conditioning activity to induce postactivation potentiation. The Journal of Strength \& Conditioning Research 21, 837-840 (2007).

Page $14 / 18$ 
59. Esformes, J. I., Cameron, N. \& Bampouras, T. M. Postactivation potentiation following different modes of exercise. The Journal of Strength \& Conditioning Research 24, 1911-1916 (2010).

\section{Figures}
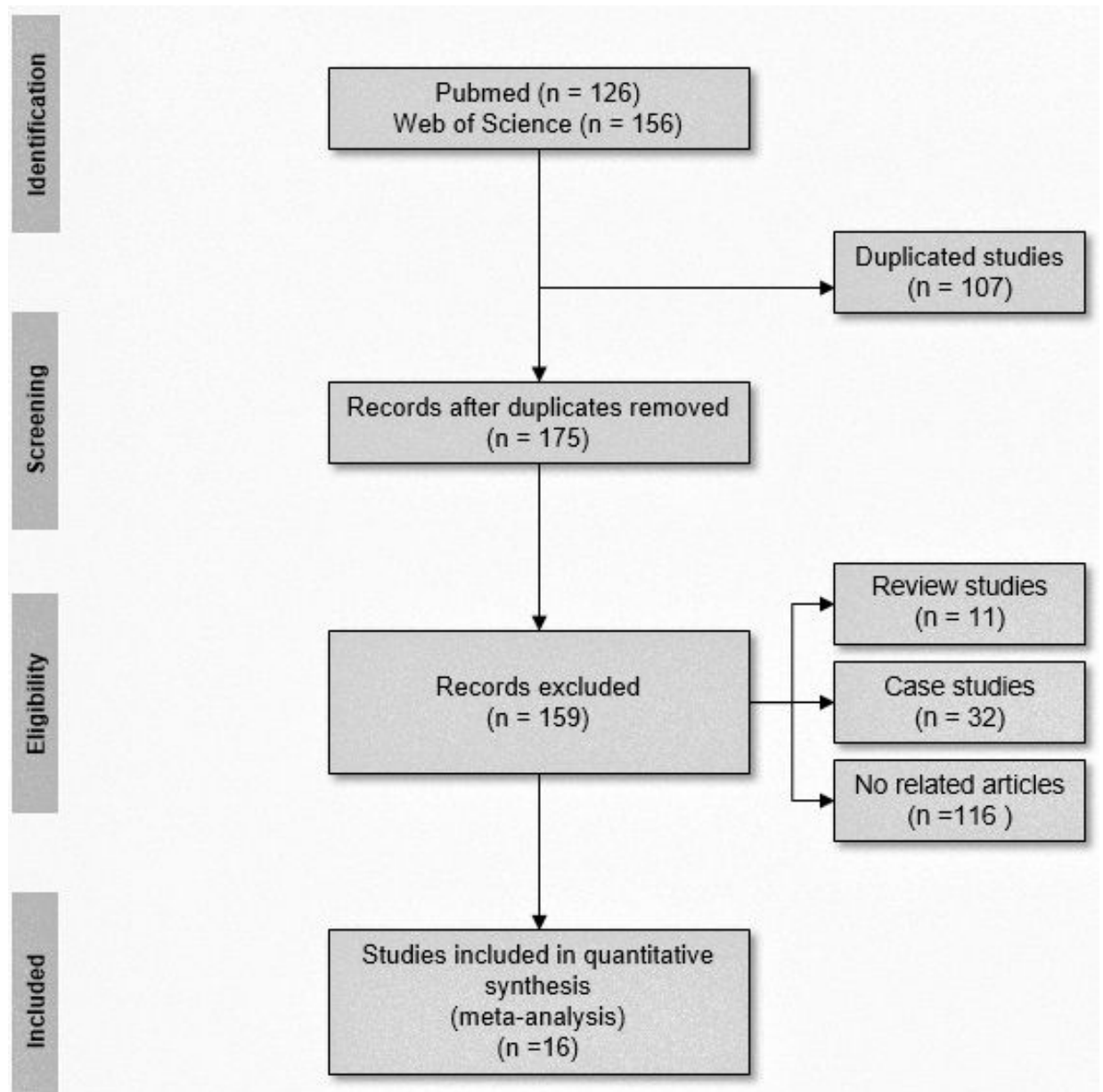

Figure 1

Search procedure and results according to PRISMA flow chart 


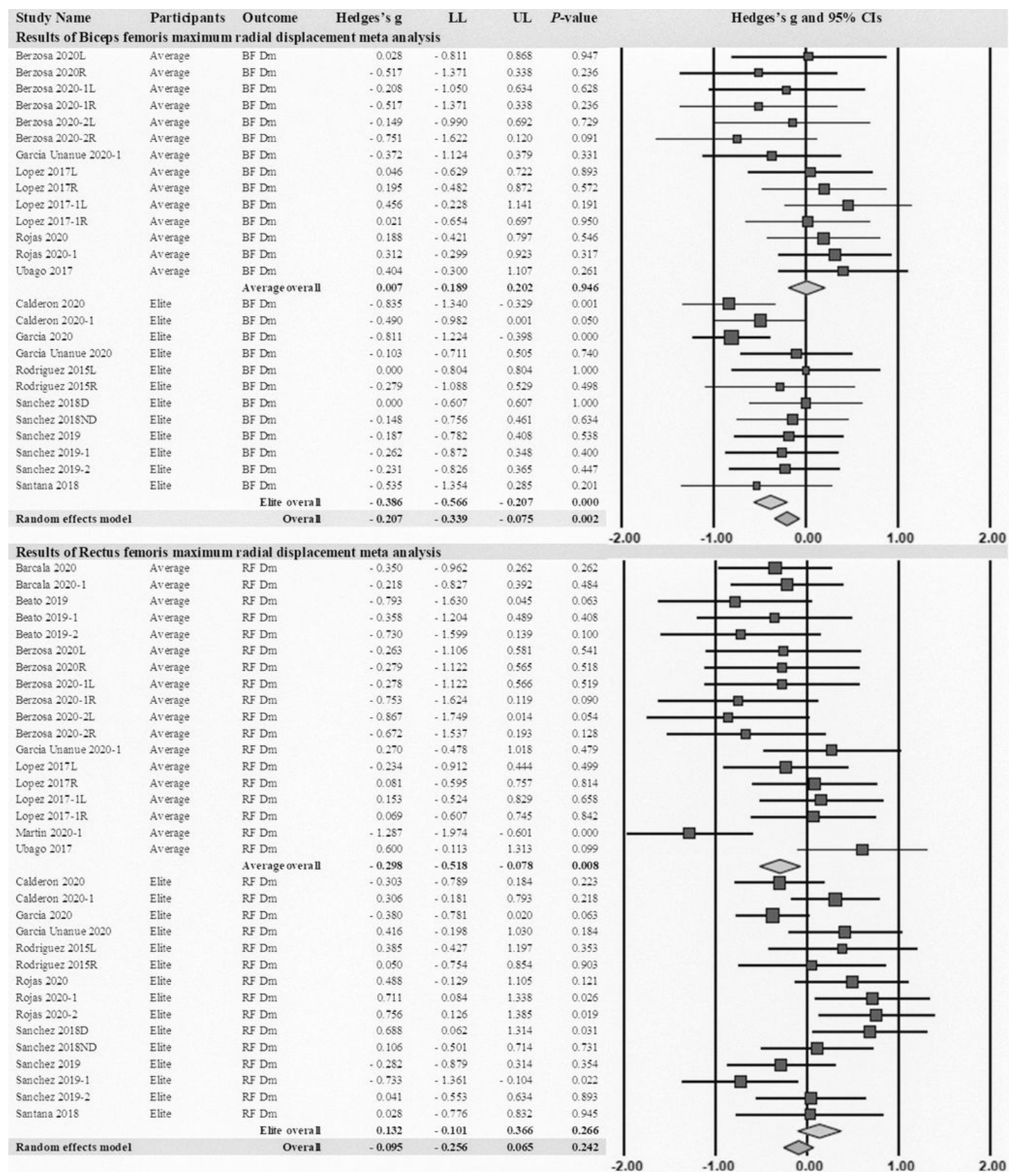

Figure 2

Results of the biceps femoris and the rectus femoris maximum radial displacement meta-analysis Caption $L$ left, $R$ right, $D$ dominant, ND non-dominant, LL lower-limit, UL upper-limit, BF Dm biceps femoris maximum radial displacement, RF Dm rectus femoris maximum radial displacement, CLs confident intervals Each size of individual effects was proportional to their weight; Diamonds indicate overall effect size 


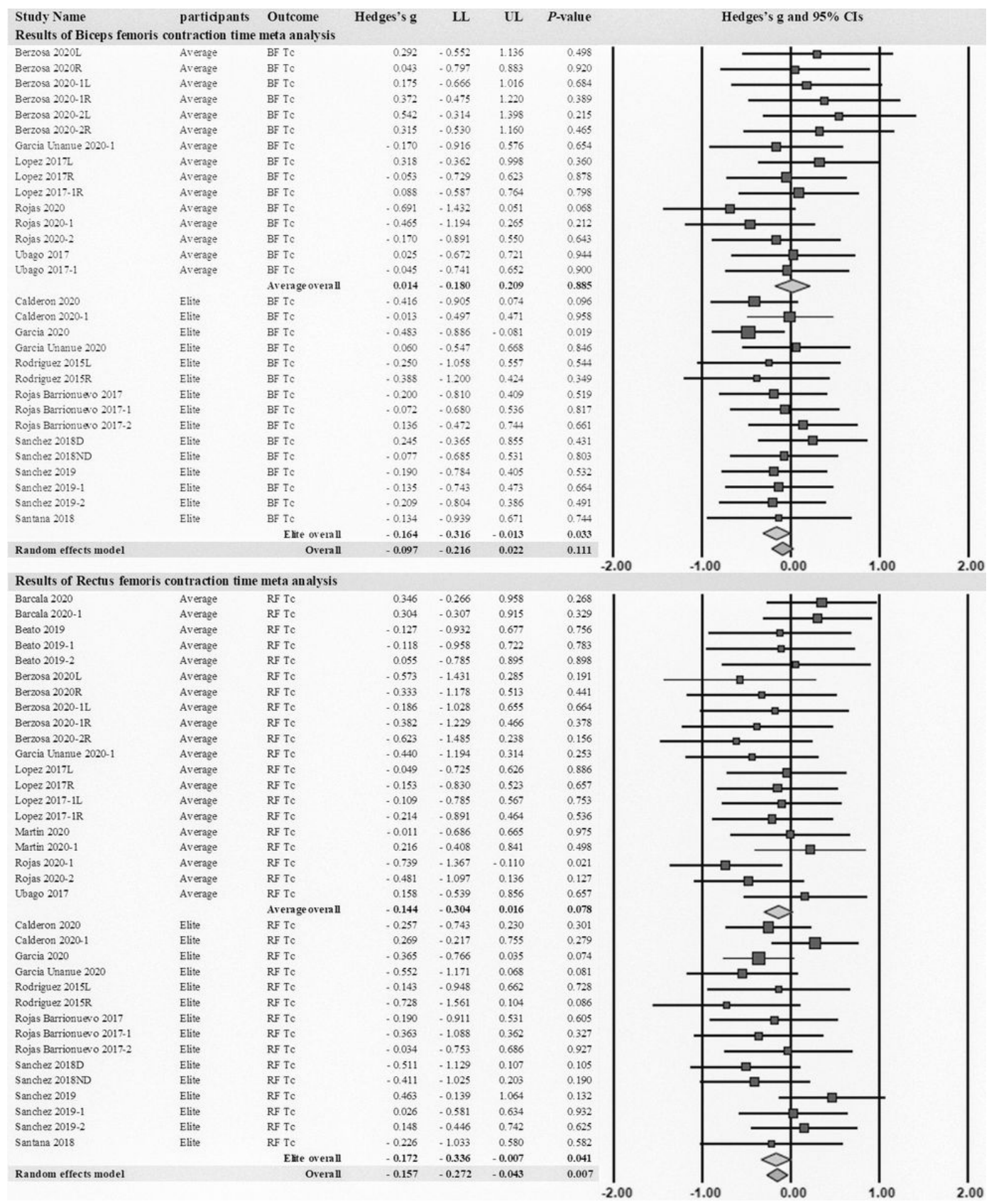

Figure 3

Results of the biceps femoris and the rectus femoris contraction time meta-analysis Caption L left, R right, D dominant, ND non-dominant, LL lower-limit, UL upper-limit, BF Tc biceps femoris contraction time, RF Tc rectus femoris contraction time, CLs confident intervals Each size of individual effects was proportional to their weight, Diamonds indicate overall effect size 


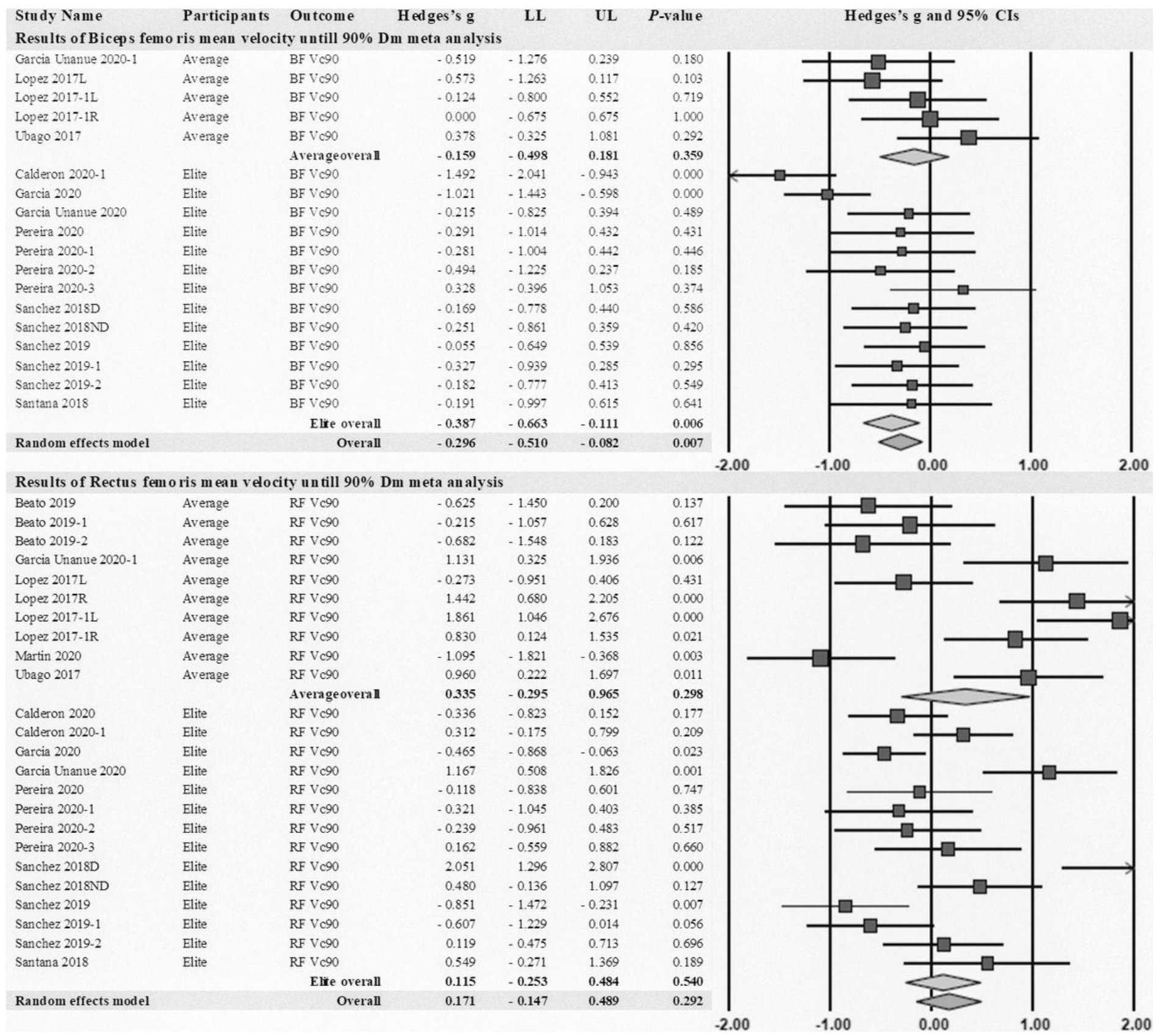

\section{Figure 4}

Results of the biceps femoris and the rectus femoris mean velocity until $90 \%$ Dm meta-analysis Caption L left, $R$ right, $D$ dominant, ND non-dominant, LL lower-limit, UL upper-limit, BF Vc90 biceps femoris mean velocity until $90 \%$ Dm, RF Vc90 rectus femoris mean velocity until $90 \%$ Dm, CLs confident intervals Each size of individual effects was proportional to their weight, Diamonds indicate overall effect size

\section{Supplementary Files}

This is a list of supplementary files associated with this preprint. Click to download.

- PRISMAchecklistTMGmetaFinal.docx 